\title{
Efeito de óleo essencial de laranja associados a fungicidas no controle de doenças foliares do trigo
}

\author{
Cezar Coradini $^{1 *}$; Fernando Piccinini ${ }^{1}$; Geovane Boschmann Reimche ${ }^{1}$; Ivan Francisco Dressler da Costa ${ }^{1}$; Sérgio \\ Luiz de Oliveira Machado ${ }^{1}$
}

${ }^{1}$ Departamento de Defesa Fitossanitária, Universidade Federal de Santa Maria (UFSM), 97105-900, Santa Maria, Rio Grande do Sul (RS), Brasil. Autor para correspondência: Cezar Coradini (cezarcoradini@yahoo.com.br)

Data de chegada: 29/07/2014. Aceito para publicação em: 20/08/2015.

$10.1590 / 0100-5405 / 2020$

\section{RESUMO}

Coradini, C.; Piccinini, F.; Reimche, G.B.; Costa, I.F.D.; Machado, S.L.O. Efeito de óleo essencial de laranja associados a fungicidas no controle de doenças foliares do trigo. Summa Phytopathologica, v.42, n.1, p.105-106, 2016.

Foi conduzido um experimento em campo na safra de 2012 com o objetivo de avaliar a contribuição do óleo essencial de laranja na performance dos fungicidas visando o controle de doenças foliares do trigo. $\mathrm{O}$ delineamento experimental foi o de blocos ao acaso em arranjo fatorial $(3 \times 5)+1$, com quatro repetições. $\mathrm{O}$ fator $\mathrm{A}$ foi composto pelos fungicidas (dose do produto comercial) epoxiconazol $\left(0,75 \mathrm{~L} \mathrm{ha}^{-1}\right)$, piraclostrobina $\left(0,80 \mathrm{~L} \mathrm{ha}^{-1}\right)$ e epoxiconazol+piraclostrobina $\left(0,75 \mathrm{~L} \mathrm{ha}^{-1}\right)$ e o fator B pelos adjuvantes (doses) óleo mineral $\left(500 \mathrm{~mL} \mathrm{ha}^{-1}\right)$ e óleo essencial de laranja $\left(0,50,100\right.$ e $\left.150 \mathrm{~mL} \mathrm{ha}^{-1}\right)$. O tratamento adicional compreendeu-se pela testemunha (água). O controle de manchas foliares na folha bandeira foi mais eficaz com a aplicação de epoxiconazol e de epoxiconazol+piraclostrobina independentemente do adjuvante usado ser óleo mineral ou óleo essencial de laranja, enquanto que os adjuvantes testados não interferiram no controle da ferrugem. O peso do hectolitro, massa de mil grãos e a produtividade do trigo não foram afetados negativamente pelas diferentes concentrações do óleo essencial de laranja.

Palavras-chave: Triticum aestivum L., manchas foliares, ferrugem da folha, adjuvantes.

ABSTRACT

Coradini, C.; Piccinini, F.; Reimche, G.B.; Costa, I.F.D.; Machado, S.L.O. Effect of orange essential oil associated with fungicides to control wheat leaf diseases Summa Phytopathologica, v.42, n.1, p.105-106, 2016.

A field experiment was conducted during the 2012 growing season with the aim of evaluating the contribution of orange essential oil to the performance of fungicides on the control of wheat leaf diseases. The experimental design was in randomized blocks, as factorial arrangement $(3 \times 5)+1$, and four replicates. Factor A was composed of the fungicides (dose of the commercial product) epoxiconazole $\left(0.75 \mathrm{~L} \mathrm{ha}^{-1}\right)$, pyraclostrobin $\left(0.80 \mathrm{~L} \mathrm{ha}^{-1}\right)$ and epoxiconazole+pyraclostrobin $\left(0.75 \mathrm{~L} \mathrm{ha}^{-1}\right)$ and factor B was composed of the adjuvants (doses) mineral oil $\left(500 \mathrm{~mL} \mathrm{ha}^{-1}\right)$ and orange essential oil $(0,50,100$ and $150 \mathrm{~mL} \mathrm{ha}^{-1}$ ). The additional treatment was represented by a control (distilled water). Flag leaf spot control was more efficient with the use of epoxiconazole and epoxiconazole+pyraclostrobin whether the used adjuvant was mineral oil or orange essential oil, while the tested adjuvants did not affect rust control. The hectoliter weight, mass of one thousand grains and wheat yield were not negatively affected by the different concentrations of orange essential oil.

Keywords: Triticum aestivum L, leaf spots, leaf rust, adjuvants.

As doenças foliares na cultura do trigo que mais ocorrem são mancha marrom (Cochliobolus sativus (Ito; Kurib.), Drechs. ex. Dastur. (anamorfo: Bipolaris sorokiniana Sacc. in. Sorok.), mancha salpicada (Septoria tritici Roberge in Desmaz. (teleomorfo: Mycosphaerella graminicola (Fuckel) J. Schöt. In Chon) e ferrugem da folha (Puccinia recôndita Rob. Ex. Desm. F.sp. tritici Heriks. \& Henn). Esse complexo de doenças geralmente causa danos no trigo de até $80 \%$ na produtividade (4). Adjuvantes são usados para modificar as propriedades físicas e biológicas das misturas de pulverização para potencializar a performance dos agrotóxicos (3). Recentemente, desenvolveu-se adjuvantes naturais baseados em óleos essenciais extraídos da casca de laranjas (Citrus spp. L.), no entanto, há carência de informações sobre sua efíciência quando associado com fungicidas para o controle de doenças.

Nesse contexto, este estudo teve por objetivo avaliar o efeito do óleo essencial de laranja associado aos fungicidas epoxiconazol, piraclostrobina e de epoxiconazol+piraclostrobina no controle de ferrugem da folha e complexo de manchas foliares e na produtividade do trigo. $\mathrm{O}$ experimento foi conduzido na safra agrícola de 2012 no município em Itaara, $\left(29^{\circ} 32^{\prime} \mathrm{S}, 53^{\circ} 45^{\prime} \mathrm{W}\right.$;), RS, Brasil utilizando um delineamento experimental de blocos ao acaso em arranjo fatorial $(3 \times 5+1)$ com quatro repetições. Cada unidade experimental foi constituída por 12 linhas de trigo (cv. Quartzo ${ }^{\circledR}$ ) espaçadas $0,17 \mathrm{~m} \mathrm{e}$ com 5,0m de comprimento. O fator A foi composto pelos fungicidas (doses) epoxiconazol (Opus $\left.{ }^{\circledR} \mathrm{SC}\right)\left(0,75 \mathrm{~L} \mathrm{ha}^{-1}\right)$, piraclostrobina (Comet ${ }^{\circledR}$ CE) $\left(0,80 \mathrm{~L} \mathrm{ha}^{-1}\right)$ e epoxiconazol+piraclostrobina (Opera $\left.{ }^{\circledR} \mathrm{SE}\right)(0,75 \mathrm{~L}$ $\left.\mathrm{ha}^{-1}\right)$ e o fator B por adjuvantes (doses): óleo mineral (Assist $\left.{ }^{(B)}\right)(500 \mathrm{ml}$ $\left.\mathrm{ha}^{-1}\right)$ e doses de óleo essencial de laranja $\left(\right.$ Orobor $\left.^{\circledR}\right)(0,50,100$ e $150 \mathrm{ml}$ $\mathrm{ha}^{-1}$ ), com tratamento adicional representado pelo controle (água). A aplicação dos tratamentos em número de duas, foram realizadas com o auxílio de um pulverizador com pressão gerada por $\mathrm{CO}_{2}$, com barra de aplicação dotada de quatro pontas XR 11002 espaçadas em 
Tabela 1. Área abaixo da curva de progresso de manchas foliares na folha bandeira do trigo em resposta a fungicidas e diferentes concentrações de óleo essencial de laranja na calda de pulverização.

\begin{tabular}{|c|c|c|c|c|c|c|}
\hline \multirow{2}{*}{ Tratamentos } & \multicolumn{6}{|l|}{ Adjuvantes } \\
\hline & $\mathrm{SA}^{4}$ & $\mathrm{OM}_{500}^{5}$ & $\mathrm{OEL}_{50}^{6}$ & $\mathrm{OEL}^{6}{ }_{100}$ & $\mathrm{OEL}^{6}{ }_{150}$ & Média \\
\hline Piraclostrobina $^{2}$ & B 22 abA & B $24 \mathrm{aA}$ & B $25 \mathrm{aA}$ & B $24 \mathrm{aA}$ & $\mathrm{B} 17 \mathrm{aA}$ & $23 \mathrm{a}$ \\
\hline Controle (água) & A 373 & & & & & \\
\hline Média & $22 \mathrm{a}$ & $16 \mathrm{a}$ & $17 \mathrm{ab}$ & $17 \mathrm{ab}$ & $14 \mathrm{~b}$ & 17,6 \\
\hline CV (\%) & 14,7 & & & & & \\
\hline
\end{tabular}

"médias seguidas pela mesma letra maiúscula nas linhas e minúscula nas colunas não diferem entre si pelo teste $\mathrm{t}(\mathrm{p} \leq 0,05)$. Médias não antecedidas pela mesma letra maiúscula diferem do controle (água) pelo teste de Dunnett $(\mathrm{p} \leq 0,05) ;{ }^{1} \mathrm{Opus}^{\circledR} \mathrm{SC} ;{ }^{2} \mathrm{Comet}^{\mathbb{B}} \mathrm{EC} ;{ }^{3}$ Opera ${ }^{\circledR} \mathrm{SE} ;{ }^{4}$ sem adjuvante; ${ }^{5}$ óleo mineral $\left(\right.$ Assist $\left.{ }^{\mathbb{B}}\right) ;{ }^{6}$ óleo essencial de laranja $\left(\mathrm{Orobor}^{\mathbb{B}}\right)$.

$0,5 \mathrm{~m}$, sendo as aplicações realizada no final do emborrachamento e no florescimento do trigo (5). As severidades das doenças foram obtidas através da porcentagem de área afetada em 25 folhas bandeira e bandeira -1 , avaliando aleatoriamente 25 folhas bandeira e 25 folhas bandeira -1 na área útil das parcelas. As avaliações foram realizadas aos 15, 22, 29 , 36 e 43 dias após a primeira aplicação (DAA). Determinou-se a massa dos grãos (13\% de umidade), peso do hectolitro (PH) e a massa de mil grãos (MMG). Os dados foram submetidos aos testes das pressuposições do modelo matemático e submetidos à análise de variância (ANOVA) $(p \leq 0,05)$. As médias foram comparadas pelo teste $t(p \leq 0,05)$ e o controle (água) foi comparada com as médias usando teste Dunnett ( $\mathrm{p} \leq 0,05)$.

A análise de variância mostrou que houve interação significativa $(\mathrm{p} \leq 0,05)$ entre os fatores estudados para área abaixo da curva de progresso de mancha foliar da folha bandeira (AACPMFb). Para AACPMFb (Tabela 1), os fungicidas epoxiconazol+piraclostrobina diferiram significativamente do tratamento sem adjuvante independentemente do adjuvante em uso (óleo mineral e óleo essencial de laranja). Nas concentrações $\left(50,100\right.$ e $\left.150 \mathrm{~mL} \mathrm{ha}^{-1}\right)$ de óleo essencial de laranja na calda de pulverização houve controle das manhas foliares, não diferindo $(\mathrm{p} \leq 0,05)$ do tratamento com óleo mineral. A diferença de eficácia de epoxiconazol+piraclostrobina com diferentes tratamentos adjuvantes, em relação ao tratamento sem adjuvante, pode ser devido ao efeito do adjuvante que segundo (2), servem para proporcionar uma maior cobertura da folhagem, promovendo uma melhor proteção. Para área abaixo da curva de progresso de ferrugem da folha bandeira (AACPFb), área abaixo da curva de progresso de ferrugem da folha bandeira ${ }^{-1}$ $\left(\mathrm{AACPFb}^{-1}\right)$ e a área abaixo da curva de progresso de mancha foliar bandeira $^{-1}\left(\mathrm{AACPMFb}^{-1}\right)$, a interação não foi significativa (dados não mostrados). A AACPFFb, $\mathrm{AACPFFb}^{-1}$ e a $\mathrm{AACPMFb}^{-1}$ não diferiram estaticamente entre os fungicidas e adjuvantes aplicados, porém mostrou melhor desempenho quando comparado a testemunha (água). Os adjuvantes testados não diferiram $(\mathrm{p} \leq 0,05)$ para o $\mathrm{PH}, \mathrm{MMG}$ e produtividade de grãos (dados não mostrados), contudo, diferenciou do controle (água). Os adjuvantes minerais podem ter efeito fitotóxico às plantas, podendo reduzir o crescimento e interferir no desenvolvimento de plantas (1), assim esses resultados mostram que a substituição do óleo mineral pelo óleo essencial de laranja (50 a $\left.150 \mathrm{~mL} \mathrm{ha}^{-1}\right)$ na calda de pulverização não altera o controle do conjunto de doenças analisadas, assim como, a PH, MMG e a produtividade de grãos de trigo.

\section{AGRADECIMENTOS}

Os autores agradecem ao apoio financeiro recebido da Coordenação de Aperfeiçoamento de Pessoal de Nível Superior (CAPES).

\section{REFERÊNCIAS BIBLIOGRÁFICAS}

1. Gao, J.; Hofstra, G.; Fletcher, R.A. Anatomical changes induced by triazoles in wheat seedlings. Canadian Journal of Botany, v.66, n.12, p.11781185,1988 .

2. Hazen, J.L. Adjuvants: terminology, classification, e chemistry. Weed Technology, Champaign, v.14, n.4, p.773-784, 2000.

3. Kudsk, P.; Mathiassen, S.K. Analysis of adjuvant effects and their interactions with variable application parameters. Crop Protection, v.26, n.3, p.328-334, 2007.

4. Mehta, Y.R.; Riede, C.R.; Campos, L.A.C.; Kohli, M.M. Integrated management of major wheat diseases in Brazil: an example for the Southern Cone region of Latin America. Crop Protection, v.11, n.6, p.517-524, 1992.

5. Zadoks, J.C.; Chang, T.T.; Konzak, C.F. A decimal code for the growth stages of cereals. Weed Research, v.14, n.6, p.415-421, 1974. 